\title{
Autorenverzeichnis 1983
}

Aigner, K., 74

Alves de Oliveira, C. R., 126

Anfreeff, M.,179

Arlin,Z., 179

Arnold, H., 62

Aul,C.,280

Aulbert,E.,82

Axt,D., 126

Berdel, W. E.,306 Bergholz,M.,84 Bernsmeier, R., 4 Beron, G., 26 Beyer, J.-H., 114 Bichler, E.,314 Bleichner, F., 58 Blum, U., 12 Boll, I., 66 Borghardt, J., 114 Bouchoris, N., 8 Boutis,L.,8

Brandt, M., 56 Breithaupt,H.,296 Bremer, K., 16 Bruhn,H.D.,4 Büchner,Th.,176,179

Caliebe,W.,114 Clarkson,B.D.,179

Dauer,W.,206 Daxecker, F., 314 Diehl,V.,306 Dimitriadis, K., 8 Drescher, J., 26 Drings,P.,62 Engelhardt,R.,188

Fibbe,W. E.,200 Fiebig,H. H., 188 Fischer, H.-P., 296 Fischer, J.Th., 280 Franke,H.,1212

Fries, R., 320 Funovics, J., 148

Gahr, M.,88 Gerhartz,H., 114 Gericke, D., 36 Germano, G., 62 Goebel, U.,26 Graubner, M.,306

Gropp, C, 306 Gutjahr,P.,26

Hartlapp,J.H.,288

Hatzigogos, K., 8

Hechtel,R.,74

Heidemann,E.,136,144

Hellriegel,K.P.,169

Нeuß,H., 188

von Heyden, H.W., 84, 88, 114

Hiddemann, W., 176,179

Hoelzer,D., 170,188

Hofmann,J., 114

Hofstetter, A.,36

Holzgrebe, E., 4

Holzner,J.H., 148

Hossfeld,D.K., 184

Hudec,M.,320

Illiger, H. J.,288

Jürgens, H., 26

Kamanabroo, D., 176 Kastenbauer, E., 114 Kehl,A.,84 Keiditsch, E., 36 Kleinmanns, G., 206

Klippel,K. F.,126 Klippstein, Th., 306 Knieí,J. J.,206 Knispel,J.,292 König,H. J.,56

Koukourikos, S., 8 Krähling,K. H.,56

Krebs,A.,121 Kubaschinski, G., 184 
Landbeck, G., 26 Lersch, Chr., 36 Link, FL, 188 Lissers,R., 114 Loo, van de, J., 176 Luck, P., 4 Meisl,F.,148 Mende,S.,58,306 Mertens,H. G.,106 Meuret, G.,58 Mitrou,P. S.,192,306

Mouratidou, D., 8 Müller,K.M.,62 Müller-Wickup, J., 26

Nagel,G. A.,84,88, 114 Niederle, N., 16 Nowrousian, M. R., 184

Obrecht,J. P.,62 Ostendorí,P.,22, 144

Peiss, J.,288 Pfeilsticker,U.,136 Platz,H.,320 Polterauer, P., 148 Pralle,H.,188,296 Preiss, J., 126

Queisser, W. , 62

Rudert,H.,114

Safarini, S., 8 Seeber, S., 16 Seuffer,R.,74 Schaefer,U. W.,184 Schärfe,T.,126 Scherff,A.,88

Scherpe, A., 114

Schmidt, C. G.,16,82,184 Schmitz, R.,188 Schneider, B., 114 Schneider, W., 280 Schöck,V.,109

Schoetensack, B., 16 Schroeder, M., 114 Schroder, M., 114 Schröter, W. , 88 Schütte, J., 16

Schulte,H.,176 Schulz,A.,296

Spaar, H.-J., 26

Steinke,B.,22,144 Stoeter, P.,58

Thiel,R.,292 Thöne, I., 26 Thomas, P., 26 Tonn,J.C.,74 Travantzis, J., 8

Ungeheuer, E., 12 Urbanitz,D., 176

Vaupel,H. A.,288 Voss,A. C.,22,136

Wacha,H., 12

Waller,H. D.,22, 136,144

Wallis,H.,292

Weber, W., 62

Westerhausen, M., 62,114, 206

Willemze, R.,200

Wilms, K., 136, 144

Winkler,K.,26

Wörmann,B., 179

Zurborn,K. H.,4 Zwaan,F. E.,200 